\title{
Evolution des infections naturelles sur les mangues, les avocats et les bananes en Côte d'Ivoire et principaux champignons responsables
}

Adjoa Marie-Joséphine KOUADIA ${ }^{*}$, Kouabenan ABO1, Kouakou Théodore KOUADIO1

${ }^{1}$ Laboratoire de Phytopathologie et de Biologie Végétale, Département de Formation et de Recherche Agriculture et Ressources Animales, Institut National Polytechnique Félix Houphouët-Boigny (INP-HB), BP 1313 Yamoussoukro $\left({ }^{*}\right)$ Auteur correspondant : E-mail : linglee11134@gmail.com

Original submitted in on 24th January 2019. Published online at www.m.elewa.org/journals/ on $28^{\text {th }}$ February 2019 https://dx.doi.org/10.4314/jab.v134i1.8

\section{RÉSUMÉ}

Objectif : Les altérations fongiques constituent une des causes majeures des pertes post récolte des fruits frais en Côte d'Ivoire. L'objectif de cette étude était d'analyser les infections naturelles qui apparaissent sur les fruits dans les principales zones de production du pays, et d'identifier les principaux agents pathogènes responsables.

Méthodologie et résultats : L'étude a consisté à la collecte, de manière aléatoire, des fruits dans les zones Centre, Sud, Est et Nord du pays, à la notation quotidienne des infections naturelles apparues sur les fruits, sur une durée de 6 à 9 jours et à l'isolement ainsi que l'identification des champignons qui leur sont associés. Les résultats ont montré une évolution du taux cumulé des infections naturelles de tous les fruits et la présence de 16 groupes morphologiques dont les plus dominants sont ceux à thalle gris $(93,75 \%)$ au niveau de la mangue, ceux à thalle noir cotonneux $(73,53 \%)$ pour l'avocat et ceux à thalle rose $(81,25 \%)$ pour la banane. L'identification a montré une diversité de champignons pathogènes dont les principaux genres sont: Colletotrichum, Fusarium, Aspergillus, Phoma, Penicillium, Curvularia, Botryodiplodia et Rhizoctonia.

Conclusion et application des résultats : Les fruits frais subissent après récolte l'impact des champignons pathogènes par les infections qu'ils causent. Ces infections augmentent au fur et mesure que les fruits murissent. Ces résultats constituent une bonne base pour le renforcement des stratégies de lutte. Toutefois, des études complémentaires, notamment pathogéniques et moléculaires des principaux champignons, sont nécessaires pour mieux les connaitre et envisager, ainsi, des méthodes intégrées de lutte plus inclusives.

Mots clés: Fruits, post-récoltes, infections, champignons pathogènes.

\section{ABSTRACT}

Objective: Fungal alterations are one of the major causes of post-harvest losses of fresh fruit in Côte d'Ivoire. The objective of this study was to analyze the natural infections that appear on fruits produced in the main production areas of the country, and to identify the main pathogens.

Methodology and results: The study consisted of random fruit collection in the main growing areas, daily recording of natural infections on the fruit over a period of 6 to 9 days and isolation of the associated fungi. 
The results showed an evolution of the cumulative rate of natural infections of all fruits and the presence of 16 morphological fungal groups of which the most dominant are those with grey thallus $(93.75 \%)$ in mango, those with cottony black thallus (73.53\%) for avocado and those with pink thallus (81.25\%) for banana. Identification showed a diversity of pathogenic fungi including the genera Colletotrichum, Fusarium, Aspergillus, Phoma, Penicillium, Curvularia, Botryodiplodia and Rhizoctonia.

Conclusion and application of results: Fresh fruits quality are influenced by pathogenic fungi through the infections they cause after harvest. These results provide a good basis for control strategy enhancement. However, additional studies, including pathogenic and molecular components of the main fungi, are needed to better understand them and thus consider more inclusive integrated control methods

Key words: Fruits, post-harvest, infections, pathogenic fungi.

\section{INTRODUCTION}

Les fruits constituent une importante source d'alimentation en vitamines et en sels minéraux (Yaouba \& Mpounze, 2017). La production mondiale de fruits est estimée à 92 millions de tonnes (Anonyme, 2017). En Côte d'lvoire, les fruits occupent une place de choix dans l'économie nationale. Ils sont, en plus, d'importantes sources de revenus pour les agriculteurs. Les fruits sont aujourd'hui classés au 5 ème rang des filières pourvoyeuses de devise (FIRCA, 2014). Les principaux fruits produits sont, en autres, l'avocat, la banane, la mangue. Les productions atteignent en moyenne par an $250000 \mathrm{~T}$ pour la banane, 150000 T pour la mangue (FIRCA, 2014) pour des superficies cultivées respectives de 7500 ha et 20 000 ha. Les vergers d'avocatiers occupent seulement 300 ha. Les principales zones productrices sont localisées dans les régions Centre et Sud pour l'avocat, la région Sud autour d'Abidjan pour la banane dessert et la région Nord pour la mangue. Initialement produits pour la consommation locale, les fruits sont pour la plupart exportés vers les marchés européens. Toutefois, la production ivoirienne de fruits est confrontée à de nombreux problèmes dont les altérations post récoltent dues à la recrudescence des agents pathogènes. Ces infestations parasitaires sont liées aux difficultés d'applications des bonnes pratiques agricoles, mais surtout à la manutention post récolte inadéquate des fruits (Yaouba \& Mpounze, 2017). Ce qui a pour conséquence les interceptions de cargaisons au niveau des marchés européens (Touré, 2012). Parmi ces maladies de conservation, les altérations fongiques constituent une des principales contraintes à la qualité des fruits frais en Côte d'Ivoire (Kouamé et al., 2011; Alloué-Boraud et al., 2015). On estime qu'environ 20 à $25 \%$ des fruits récoltés sont décomposés par des agents pathogènes au cours de la manipulation post récolte (Al-Hindi et al., 2011). Les contaminations des fruits par les infections fongiques peuvent se produire depuis le champ ou lors des opérations de conditionnement après la récolte, en entrepôt et parfois après l'achat par le consommateur (Coleacp, 2011). Leur développement est favorisé par le processus de murissement des fruits. Ces pourritures affectent la valeur marchande des fruits et engendrent des pertes pouvant aller jusqu'à 90\% (Anonyme, 2013). La diversité de pathogènes à l'origine de ces affections rend difficile leur contrôle. En effet, les méthodes de lutte chimiques employées pour leur réduction posent les contraintes de la résistance grandissante des agents pathogènes (Spore, 2016) et celles des limites maximales de résidus de pesticides dans les fruits imposés, au niveau des pays importateurs. De nombreuses études ont été entreprises dans certains pays comme le Pakistan, l'Inde, le Sénégal, le Cameroun afin d'identifier les agents pathogènes associés aux altérations post récolte des fruits (Diedhiou et al., 2007 ; Djeugap et al., 2009 ; Alemu, 2014 ; Djeugap et al., 2015 ; Malik et al., 2016). En Côte d'Ivoire, les travaux de Kouamé et al. (2011), N'Guetta et al. (2013) ont permis de mettre en évidence la diversité morphologique et pathogénique des souches de Colletotrichum sp, un agent de pourriture post récolte, causant la maladie de l'anthracnose sur mangue. En 2017, Kouadio et al., ont mené une 
étude similaire qui a permis d'inventorier les champignons pathogènes associés maladies post récolte des bananes desserts et proposer une méthode de lutte par l'emploi de l'Azoxystrobine. Cependant, peu d'études ont été envisagées sur l'état sanitaire actuel des fruits, avec une vision croisée sur l'avocat, la banane et la mangue, prenant en compte les principales zones de production de chacun de ces fruits. L'objectif de la présente étude est de contribuer à une gestion post récolte des infections des fruits par une meilleure connaissance de celles-ci ainsi que les champignons en cause.

\section{MATÉRIEL ET MÉTHODES}

Matériel végétal : Le matériel végétal est constitué de l'avocat, en provenance des régions Centre et Sud, de la banane provenant de la région Sud autour d'Abidjan et de la mangue en provenance des régions Centre et Nord (Figure 1). Au total, 175 bananes, appartenant

aux variétés Grande Naine et William du Sous-groupe Cavendish, ont été collectées. Pour la mangue, 185 fruits, appartenant aux variétés Amélie, Kent, Keith, Brook et variétés locales, ont été échantillonnés. Enfin, 307 avocats tout-venants ont été collectés.

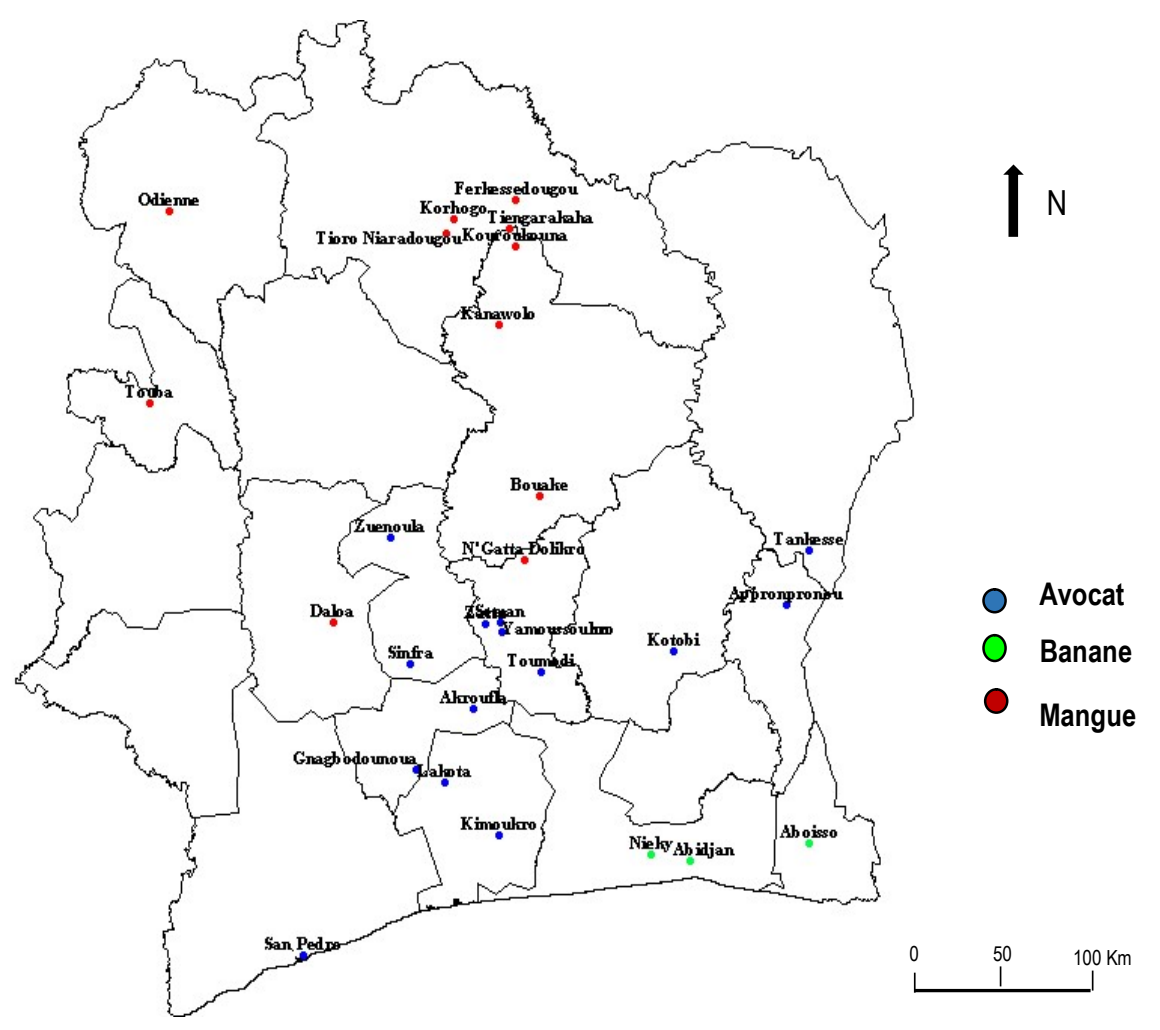

Figure 1: Zones de collecte des fruits d'avocats, de banane et de mangue de juillet 2016 à avril 2018

Matériel technique: Les études en laboratoire ont nécessité différents appareils classiques et consommables d'usage en pathologie végétale. Entre autres, il s'est agi de: une hotte à flux laminaire, un autoclave, des étuves, un bec bunsen, la verrerie, des milieux de culture.

Méthodes

Collecte des fruits: Les fruits ont été collectés à maturité physiologique dans les plantations des différentes zones de production des fruits et sur les marchés locaux et ce par vague selon leur disponibilité. Ainsi, l'avocat a été collecté pendant sa saison de production, de mars à septembre en 2017 et 2018 (Tableau 1) dans les départements de Yamoussoukro, Oumé, Gagnoa, Sinfra, Toumodi, Bouaflé, Koun-Fao, Akoupé, Abengourou, Arrah, San-pédro et Zuénoula. Quant à la collecte des échantillons de mangue, elle a été réalisée de mars 2017 à juillet 2017 et de février 
2018 à avril 2018 dans les départements de Daloa, Korhogo, Bouaké, Touba, Yamoussoukro, Ferkéssédougou, Niakaramandougou, Abengourou et Odiénné. Les échantillons de banane provenaient des plantations industrielles de la Société de Culture Bananière (SCB), Songon-Niéky, Attinguié, Aboisso Domaine Elima et Akressi Ayamé, de juillet 2016 à Avril 2017 (Tableau 1).

Tableau 1 : Localisation des zones de collecte des variétés de fruits en Côte d'Ivoire de juillet 2016 à avril 2018

\begin{tabular}{|c|c|c|c|c|}
\hline \multicolumn{2}{|c|}{ Provenances } & $\begin{array}{c}\text { Date de } \\
\text { réception au labo }\end{array}$ & $\begin{array}{l}\text { Nombre } \\
\text { de fruits }\end{array}$ & Variétés \\
\hline BANANES & 5 localités & $\begin{array}{c}30 \text { juillet } 2016 \text { au } 24 \\
\text { avril } 2017\end{array}$ & 175 & Cavendish \\
\hline AVOCATS & 12 localités & $\begin{array}{c}22 \text { mars au } 03 \text { juillet } \\
2017\end{array}$ & 307 & Cultivars tout venant \\
\hline MANGUE & 11 localités & $\begin{array}{c}21 \text { mars } 2017 \text { au } 20 \\
\text { Avril } 2018\end{array}$ & 185 & $\begin{array}{l}\text { Amélie, Kent, Keitt, Brook et diverses } \\
\text { variétés locales }\end{array}$ \\
\hline
\end{tabular}

Les fruits collectés ont été acheminés au laboratoire et mis dans des bacs et disposés à la température ambiante. Les dates de réception des fruits ont été notées ainsi que le nombre de fruits et le suivi de leur état sanitaire. Dans le cas de la banane, avant cette étape de suivi sanitaire, 10 mains de banane ont été sélectionnées de manière aléatoire dans chacun des échantillons 1 (E1), Echantillon 2 (E2) et Echantillon 3 (E3). Parmi ces 10 mains de banane, une sélection aléatoire de 30 bananes a été faite et réparties en 3 lots de 10 bananes. Le dispositif expérimental est un bloc de Fisher disposé trois répétitions, la population élémentaire étant constituée par les 10 bananes. Ces bananes ont été lavées par la suite à l'eau savonneuse et rincées dans de l'eau distillée stérile avant d'être disposées dans des bacs. Le même dispositif expérimental a été appliqué à des bananes non lavées.
Les échantillons non lavés ont été étiquetés: E2nl (Echantillon 2), E3nl (Echantillon 3), E4nl (Echantillon 4) et E5nl (Echantillon 5). Elles ont été par la suite disposées dans des bacs et laissées à la température ambiante $\left(28^{\circ} \mathrm{C}\right)$. Afin d'harmoniser le murissement, les échantillons de bananes sélectionnés ont été plongés dans une solution d'éthylène à raison de $2 \mathrm{ml} / \mathrm{I}$ d'eau distillée stérile.

Détermination du nombre d'infections naturelles: Le suivi des infections naturelles a été fait par des observations quotidiennes des fruits sur une durée de 6 à 9 jours, jusqu'à leur maturité complète. Les observations des taches ou des pourritures à la surface des fruits ont permis de suivre l'évolution des infections naturelles et de déterminer leurs taux par provenance. Ce taux a été obtenu par le cumul des moyennes journalières d'infections naturelles.
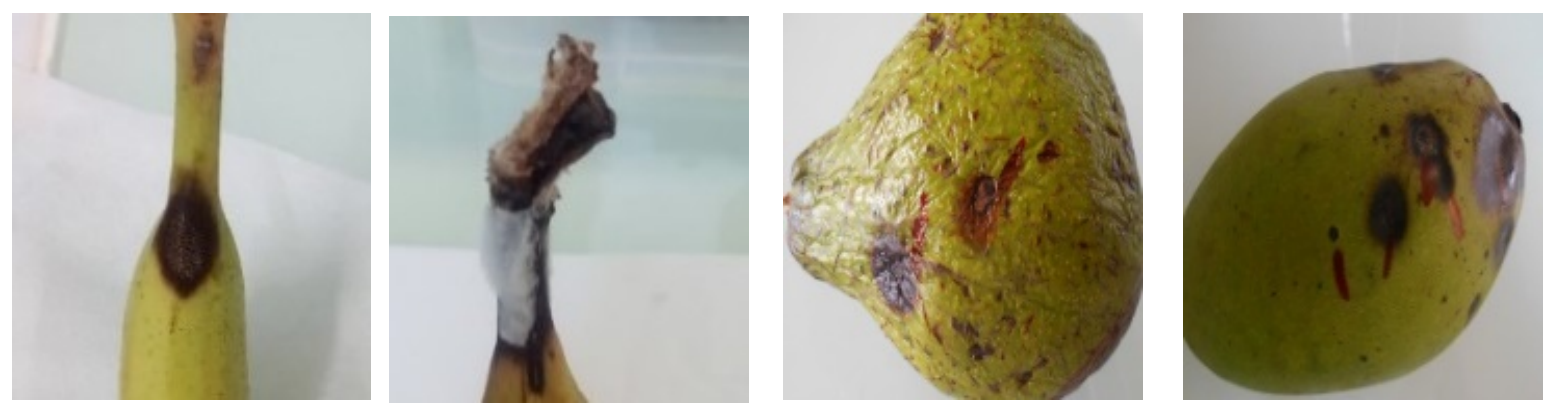

Figure 2 : Infections naturelles sur pédoncule de banane, flanc d'avocat, flanc et pédoncule de mangue

Isolement et identification des agents pathogènes : Les fruits, présentant à leur surface des taches translucides, brunes, noirâtres (Figure 2) ont été désinfectés superficiellement à l'aide d'un coton imbibé d'alcool à $70^{\circ} \mathrm{C}$, sous une hotte, dans des conditions aseptiques. Des fragments de tissus ont été ensuite prélevés à l'aide d'un scalpel au niveau du front de croissance des lésions et déposés aseptiquement sur un milieu PDA (Potato Dextrose Agar) coulé dans des boîtes de pétri de $90 \mathrm{~mm}$ de diamètre. Les boîtes ainsi ensemencées ont été mise à l'incubation dans une étuve à la température de $27^{\circ} \mathrm{C}$ pendant 3 jours. Le mycélium développé autour des fragments de tissus a été prélevé et repiqué aseptiquement de manière 
individuelle dans de nouvelles boîtes de pétri contenant du milieu PDA. Après plusieurs repiquages successifs, les cultures pures de champignons ont été obtenues puis conservées sur milieu PDA. L'identification des champignons a été réalisée sur la base de leurs caractères culturaux et morphologiques ainsi que microscopiques observés au microscope optique, en référence à des clés de détermination (Barnett \& Hunter, 1972 ; Botton et al., 1990).

Fréquence d'apparition des champignons: La Fréquence d'apparition des champignons a permis d'analyser la répartition des faciès des groupes fongiques. La Fréquence d'apparition a été obtenue selon la formule de Dédi et al. (2015) :

\section{RÉSULTATS}

Evolution des infections naturelles par fruits : La majorité des fruits ont présentés des infections naturelles. Ces infections apparaissent sous forme de points ou taches noires, brunes, translucides à la surface des fruits ou de pourritures pouvant affecter les tissus internes des fruits. Elles surviennent au niveau de l'extrémité supérieure, sur le corps (flanc) ou sur la partie distale des fruits. Les résultats ont montré une évolution de ces infections naturelles durant la période de notations sur les trois (3) types de fruits.

Infections naturelles sur avocat : Sur les échantillons d'avocats analysés, il y a une évolution du taux
$F A=\frac{\text { NAGF }}{\text { NTATF }} \times 100$

$F A=$ Fréquence d'apparition $;$ NAGF $=$ Nombre d'Apparition d'un Groupe Fongique; NTATGF = Nombre Total d'Apparition de Tous les Groupes Fongiques.

Analyse statistique: Les données obtenues (taux d'infections naturelles) ont fait l'objet d'une analyse de variance (ANOVA) à un facteur de classification à l'aide du logiciel STATISTICA version 7.1. En cas de différence significative, la comparaison des moyennes a été réalisée, au seuil de $5 \%$, à l'aide du test de Newman Keuls.

d'infections naturelles durant la période d'observation (Figure 3). L'analyse de la variance des infections naturelles des avocats a permis de mettre en évidence l'existence d'une différence significative $(P=0,016)$ entre les échantillons des différentes localités. La comparaison des moyennes cumulées a révélé que les localités de Toumodi, Akoupé, Abengourou et SanPédro présentent les plus grandes infections naturelles. Ces infections sont significativement supérieures à celles des localités de Sinfra, Koun-Fao et Zuénoula.

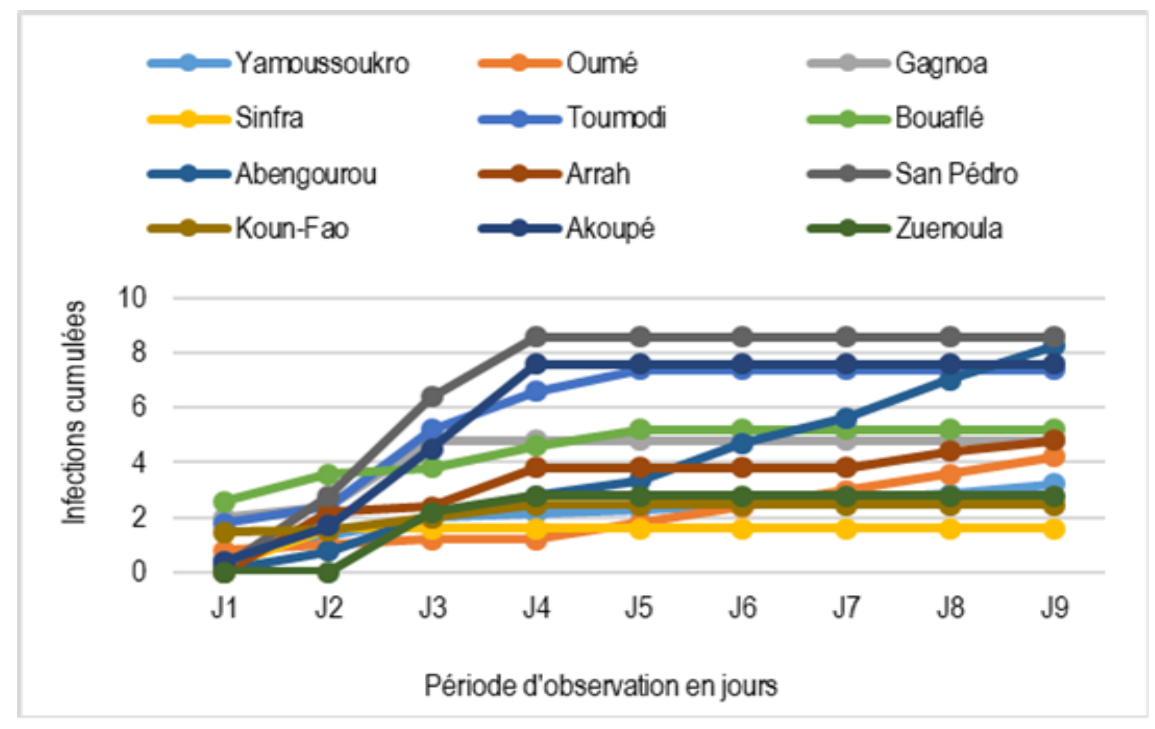

Figure 3 : Nombre moyen d'infections journalières cumulées sur fruits d'avocat 
Infections naturelles sur banane: Les infections moyennes naturelles cumulées montrent que les échantillons non lavés (E2 $\mathrm{nl}$; E3 $\mathrm{nl}$; E4 $\mathrm{nl}$ et E5 $\mathrm{nl}$ ) présentent des taux d'infections naturelles élevées par rapport aux échantillons lavés (E1; E2 et E3) (Figure
4). Les infections naturelles de bananes provenant des localités de Songon-Niéky (E2), Attinguié (E3) et Bana Comoé (E1) présentent des infections proches, significativement inférieures $(P=0,0001)$ à celles des localités d'Aboisso (E4) et Ayamé (E5).

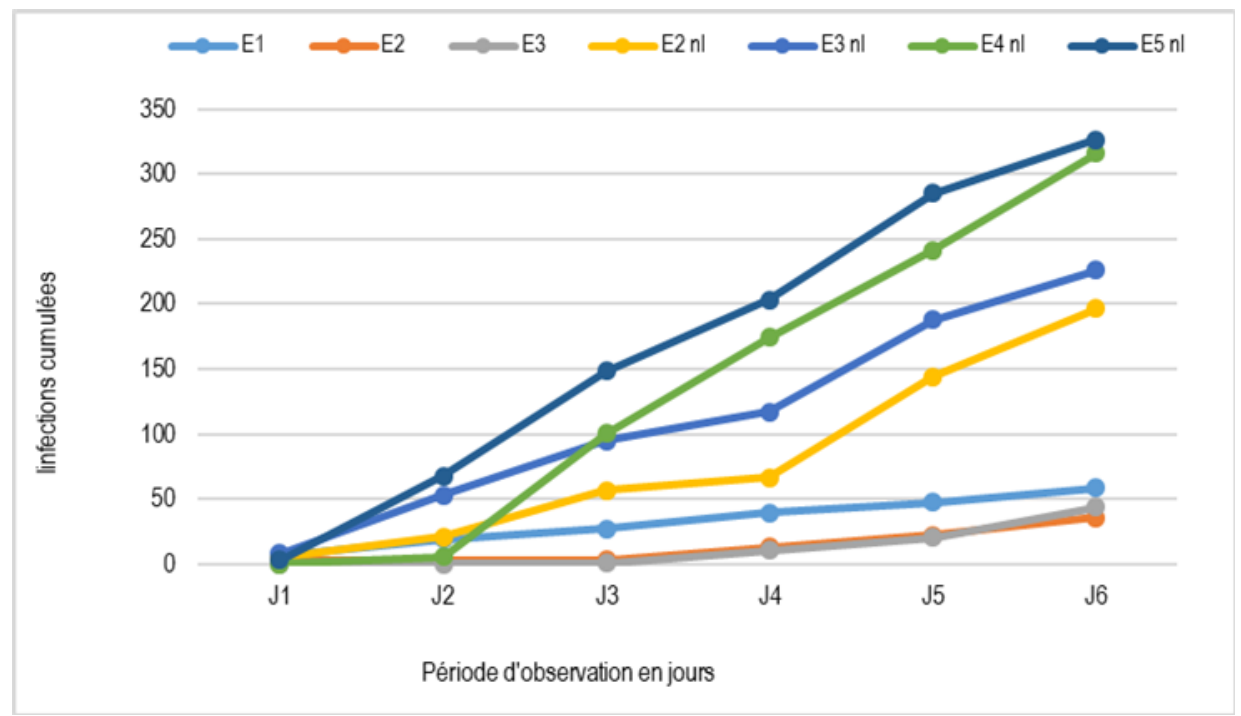

Figure 4 : Nombre moyen d'infections journalières cumulées sur fruits de banane

Infections naturelles sur mangue: L'analyse des échantillons de mangues, a également montré une évolution des infections naturelles (Figure 5) qui est plus importante dans les échantillons en provenance de Daloa, Yamoussoukro et Abengourou. Ces infections faibles au 1er jour ont augmenté progressivement jusqu'au gème jour d'observation. Les analyses statistiques ont montré qu'il y avait une différence hautement significative du 2ème jour d'observation au gème jour $(P=0,0001)$. La comparaison des moyennes a révélé que les plus faibles infections naturelles, ont été enregistrées dans la région nord à savoir Tiengarakaha, Kouroukouna, Kanawolo, Ferkessédougou et Korhogo. Ces infections sont significativement inférieures à celles de Daloa.

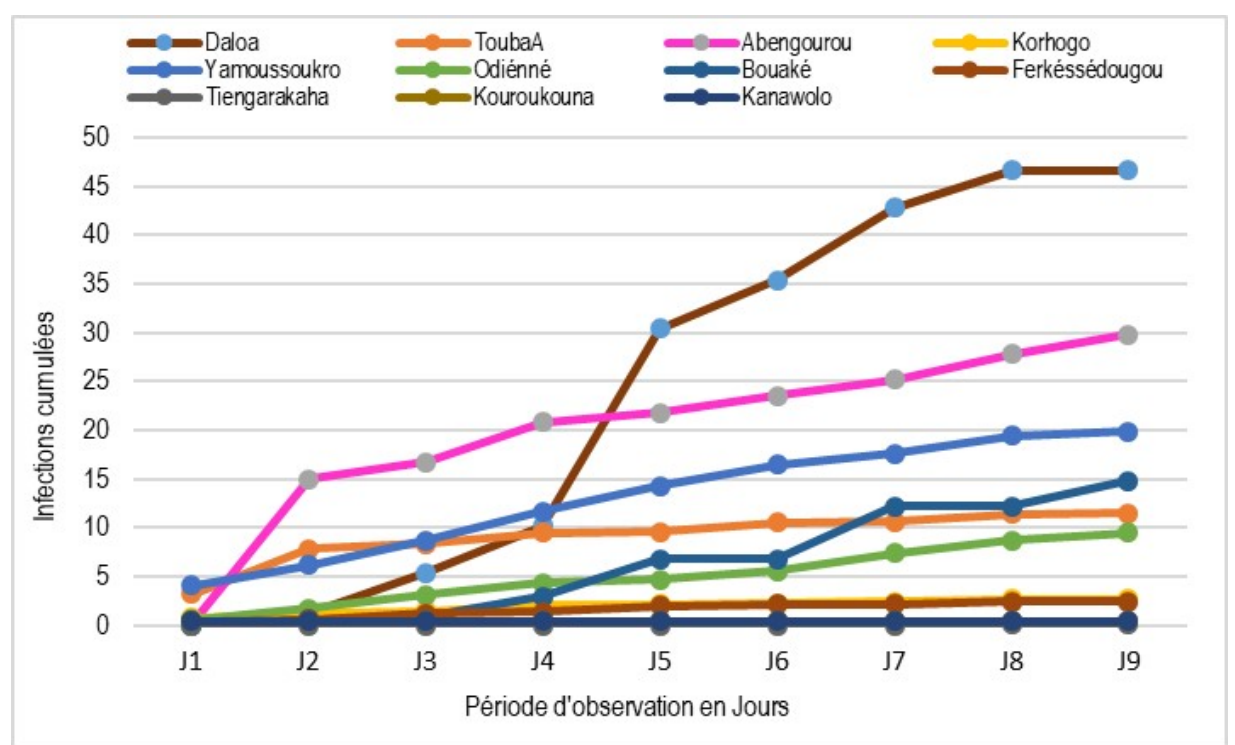

Figure 5 : Nombre moyen d'infections journalières cumulées sur fruits de mangue 
Fréquence d'apparition des groupes fongiques: Les isolats d'agents pathogènes obtenus et identifiés ont permis de montrer une diversité de pathogènes fongiques post récolte. Sur les fruits de bananes, les faciès fongiques dominants sont ceux à thalle rose orangés $(81,25 \%)$, violets $(68,75 \%)$, gris $(56,25 \%)$ et blanc $(62,5 \%)$ (Tableau 2). Sur les mangues, les faciès dominants sont ceux à thalle gris $(93,75 \%)$ et noir cotonneux $(93,75 \%)$. Ils sont suivis des thalles noir poudreux $(75 \%)$, des thalles bruns $(68,75 \%)$, et des thalles blanc floconneux $(62,50 \%)$. Sur les avocats, les pathogènes à thalle noir cotonneux sont les plus dominants avec une fréquence d'apparition de $73,53 \%$, suivis de ceux à thalle brun cotonneux $(50 \%)$, blanc floconneux et gris $(41,18 \%)$.

Tableau 2 : Fréquence d'apparition des groupes fongiques issus des échantillons d'avocat, de banane et de mangue

\begin{tabular}{c|c|c|c|c|c|c|c|c|c|c|c|c|c|c|c|c}
\hline & \multicolumn{10}{c}{ Aspects culturaux des groupes fongiques } \\
\hline & TNC & TNP & TBF & TBR & TG & TBrC & TBrP & Tbe & TVC & TVP & TR & TVi & TVeC & TVeP & Tja & Tor \\
\hline Mangues & $\mathbf{9 3 , 7 5}$ & $\mathbf{7 5 , 0 0}$ & $\mathbf{6 2 , 5 0}$ & 31,25 & $\mathbf{9 3 , 7 5}$ & $\mathbf{6 8 , 7 5}$ & 25,00 & 0,00 & 50,00 & 0,00 & 0,00 & 0,00 & 12,50 & $\mathbf{1 2 , 5 0}$ & 6,25 & 6,25 \\
\hline Avocats & $\mathbf{7 3 , 5 3}$ & 8,82 & $\mathbf{4 1 , 1 8}$ & 5,88 & $\mathbf{4 1 , 1 8}$ & $\mathbf{5 0 , 0 0}$ & 0,00 & 0,00 & 23,53 & 2,94 & 2,94 & 2,94 & 0,00 & 0,00 & 0,00 & 0,00 \\
\hline Bananes & 6,25 & 0 & $\mathbf{6 2 , 5}$ & 0 & $\mathbf{5 6 , 2 5}$ & 18,75 & 0 & 0 & 18,75 & 0 & $\mathbf{8 1 , 2 5}$ & $\mathbf{6 8 , 7 5}$ & 0 & 0 & 0 & 0 \\
\hline
\end{tabular}

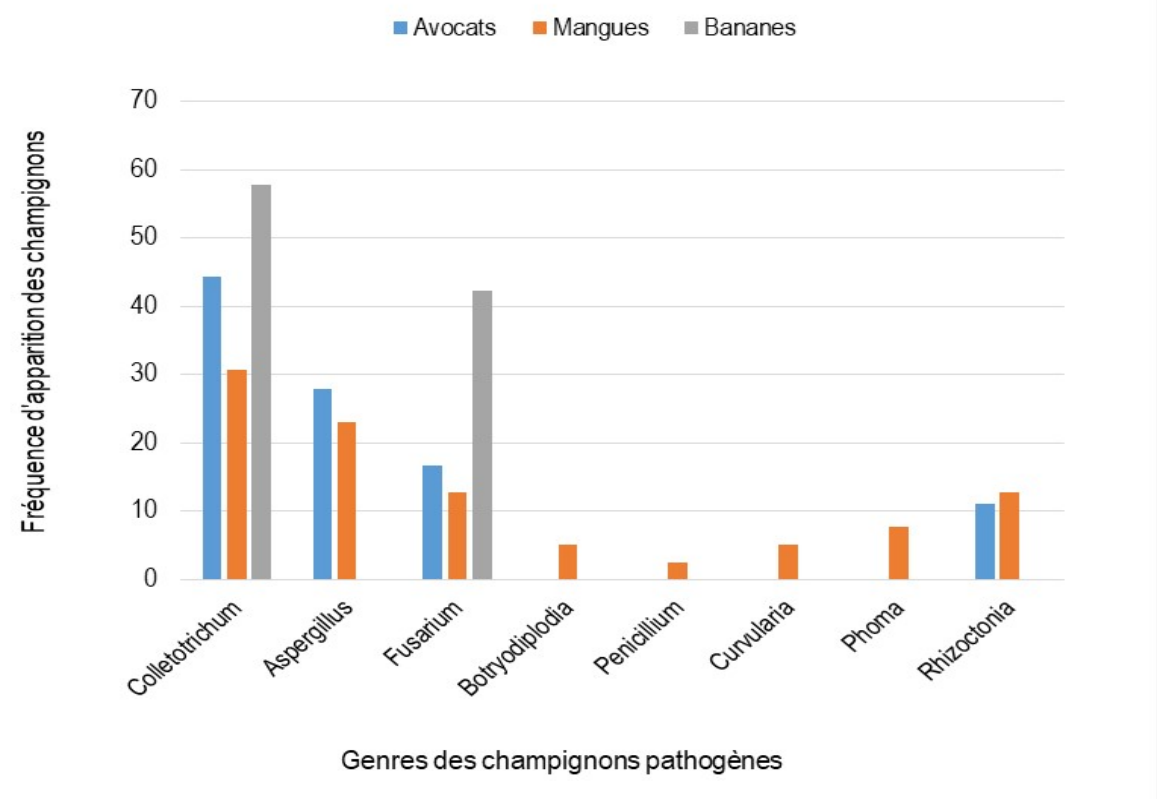

Figure 6 : Répartition des champignons pathogènes isolés dans les fruits d'avocat, de banane et de mangue

La répartition des champignons pathogènes (Figure 6) montre que les genres Colletotrichum (Figure 7), Fusarium (Figure 8), Aspergillus (Figure 9) infectent tous les fruits alors que les genres Phoma, Penicillium, Curvularia et Botryodiplodia (Figure 10) sont observés uniquement dans les mangues. Quant au genre Rhizoctonia, il infecte les échantillons d'avocats $(11,1 \%)$ et de mangues $(12,82 \%)$. Le genre
Colletotrichum a été isolé dans les bananes $(57,7 \%)$, dans les avocats $(44,4 \%)$ et dans les mangues $(30,77 \%)$. Le genre Fusarium a montré une fréquence de $16,7 \%, 12,82 \%$ et $43,30 \%$ respectivement sur avocat, mangue et banane. La fréquence d'isolement du genre Aspergillus est de $27,8 \%$ pour l'avocat et $23,07 \%$ pour la mangue. 
a
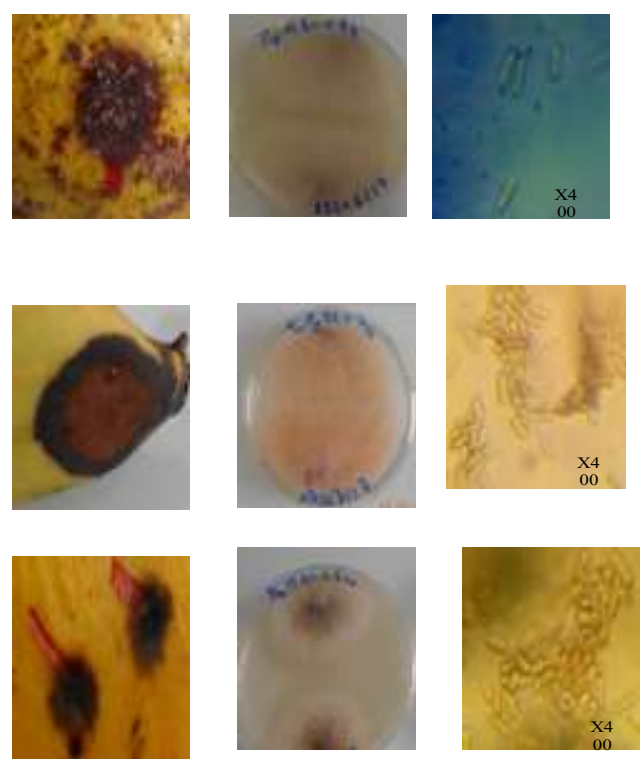

Figure 7 : Infections naturelles due à Colletotrichum sur fruits. a : Taches brune noirâtre (Infections naturelles); $\boldsymbol{b}$ : Les pathogènes responsables isolés sur milieu PDA; $\boldsymbol{c}$ : Vues au microscope des agents pathogènes
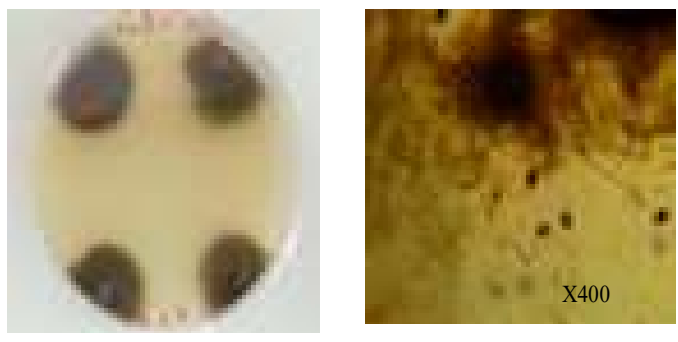

Figure 10: Infections due au genre Botryodiplodia

\section{DISCUSSION}

Evolution des infections naturelles et expression des agents pathogènes : La sensibilité des fruits aux attaques des champignons résulte de leur grande périssabilité (Kouadio et al., 2017). Ces infections apparaissent au niveau de l'extrémité supérieure (pédoncule), sur le corps (flanc) et partie distale des fruits. Ceci pourrait s'expliquer par le fait de la présence de divers pathogènes à la surface des fruits (FAO, 2004) qui sont capables de pénétrer dans les fruits par les lenticelles, les chocs ou blessures, en brisant la cuticule des fruits (Prusky \& Lichter, 2007). Les infections naturelles observées dans la grande majorité des échantillons de fruit, faible au départ augmentent,

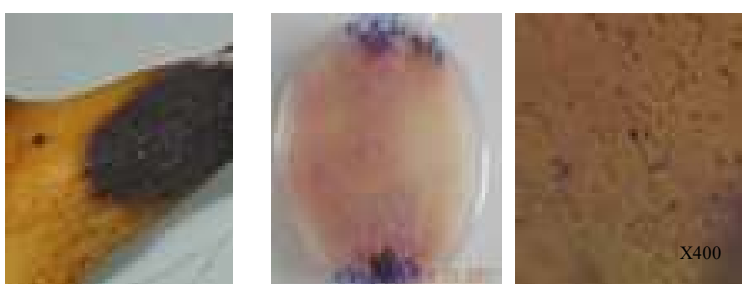

Figure 8 : Infection naturelle due au genre Fusarium
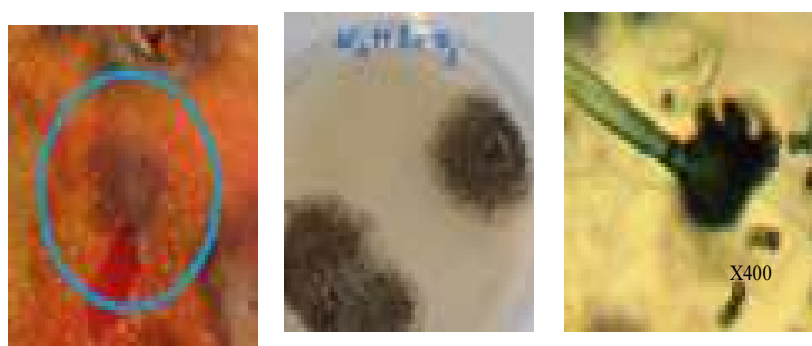

Figure 9 : Infections naturelles due au genre Aspergillus

évoluent dans le temps. Cette évolution pourrait s'expliquer par le fait que l'épicarpe des fruits est une barrière naturelle contre les agents pathogènes (Prusky \& Lichter, 2007). II ne permet pas l'entrée des champignons dans les fruits. Mais, celui-ci se fragilise, perd de sa résistance lorsque le fruit murit (Telemans, 2012). Ce constat est corroboré par Kader et Yahia (2011) et Singh et al. (2017) qui ont rapporté que les fruits sont plus sensibles aux infections des pathogènes au début de leur maturation et de leur senescence. Certains de ces champignons présents à la surface de fruits peuvent être sous forme de spores pouvant germer ou de mycélium rester inactifs (Singh et al., 
2017) et ne se révéler qu'après la récolte (Vannière et al., 2004). Les variations des charges fongiques observées dans les échantillons de mangue et d'avocat dans cette étude pourraient être attribuées aux différences de niveau d'assainissement dans la manutention et des environnements des zones de culture (Djeugap et al., 2015). Diedhiou et al. (2007); Malik et al. (2016) ont aussi montré dans leur étude qu'un verger assainis en termes de nettoyage et élagage a ses fruits moins sujets à des pourritures. De plus, la présence de stations de conditionnement dans certaines zones de production (Korhogo, Ferkessédougou et Sinémentiali) dans le cas de la mangue (Touré, 2012; FIRCA, 2014) pourrait aussi corroborer le faible niveau d'infestation observé dans certaines localités du nord. D'autres facteurs avant et après la récolte peuvent également influer sur la gravité de l'attaque des pathogènes et le développement des infections, comme la température, l'humidité relative et l'eau (Malik et al, 2016). Ainsi une humidité relative supérieure à $95 \%$ et une température comprise entre 10 et $30^{\circ} \mathrm{C}$ sont très favorables pour la germination des spores et la formation des appressoria (PIP, 2013). L'eau (la pluie) est à l'origine de la dispersion des conidies des champignons responsables de la pourriture des fruits. Singh et al. (2017) ont montré que lorsque la pluie se prolonge près du moment de la récolte, cela entraine la pourriture des fruits entreposés. De même, la rosée abondante, l'irrigation, la pluie légère et le temps pluvieux favorisent la production, la dispersion et l'infection des conidies des champignons (PIP, 2013 ; Diallo, 2016). La saison des pluies dans la région Nord commence en avril et prend fin en octobre, celle de la région du sud, commence en avril pour prendre fin en juillet (Kaman, 2013) et au centre, de mars à juin (Aquastat, 2005). Ceci pourrait expliquer les forts taux d'infections naturelles des échantillons de Daloa (collecté en juin), d'Akoupé et Abengourou (en mars), de Toumodi en mai et San-Pédro (juin). Le faible niveau d'infection enregistré dans les échantillons de bananes lavés pourrait s'expliquer par le fait que le lavage contribuerait à réduire quelque peu le nombre d'agents pathogènes présents à la surface des fruits.

\section{CONCLUSION}

Cette étude a permis d'avoir des informations sur l'étendue des infections naturelles associées aux avocats, mangues et bananes des différentes régions de production de la Côte d'Ivoire. Elle a révélé les principaux champignons pathogènes responsables. Ainsi plusieurs genres fongiques (Colletotrichum,
Diversité des principaux champignons isolés sur les fruits: Sur la base des caractéristiques des fructifications sur milieu PDA, divers champignons pathogènes attaquant les différents fruits après récolte ont été observés. Dans cette étude, les champignons les plus rencontrés sont les genres Colletotrichum, Fusarium, Aspergillus, Phoma, Penicillium, Curvularia, Botryodiplodia et Rhizoctonia, Ces résultats concordent avec ceux de Awa et al. (2012) qui ont mis en évidence une diversité de champignons, notamment Colletotrichum gloeosporioides, Botryodiplodia theobromae et Aspergillus niger associés à la pourriture des fruits de mangues mûres. Par ailleurs, les genres Colletotrichum, Fusarium, Aspergillus, Botryodiplodia et Phoma ont été signalés sur les fruits d'avocats et de mangues (Diedhiou et al., 2007 ; Djeugap et al., 2009 ; Djeugap et al., 2015). Ces champignons sont réputés être à l'origine de la détérioration de nombreux produits comme les fruits, les graines, les légumes et des tubercules aussi bien au champ qu'au cours du stockage (Dongmo et al., 2017). En outre, il a été observé dans cette étude la forte prévalence du genre Colletotrichum sur les trois fruits. Cela pourrait s'expliquer par le fait que c'est un champignon qui est capable de coloniser plusieurs hôtes (mangue, banane, orange, avocat, goyave, papaye, safou, citron vert acide, pomme, grenade, etc.) et affecter toutes les parties de la plante, ainsi que ses fruits (Lakshmi et al., 2011; Diallo, 2016). II a été aussi signalé que ce pathogène affecte les fruits, cause des maladies sur les fruits immatures et en croissance dans les conditions de plein champ, et endommage les fruits pendant le transport et l'entreposage (Wharton et al., 2004). Le genre Fusarium est le deuxième champignon le plus rencontré dans cette étude car il affecte également les trois fruits. II colonise le plus souvent l'extrémité des fruits (banane, mangue), mais également le flanc des fruits (Kouadio et al., 2017). Le genre Aspergillus est couramment rencontré sur divers fruits lors de leur stockage et plusieurs travaux ont montré l'implication d'A. niger dans la détérioration de nombreux fruits et légumes (Yaouba \& Mpounze, 2017). Tous ces champignons causent des maladies post récolte.

Fusarium, Aspergillus, Phoma, Penicillium, Curvularia, Botryodiplodia et Rhizoctonia) sont à l'origine des expressions de ces infections naturelles qui se manifestent sur toutes les parties des fruits étudiés, surtout lors de leur maturation. Les résultats suggèrent la nécessité d'élaborer des stratégies de gestion de ces 
pathogènes, sur la base de leur importance relative et

\section{RÉFÉRENCES BIBLIOGRAPHIQUES}

Al-Hindi RR, Al-Najada AR, Mohamed SA, 2011. Isolation and identification of some fruit spoilage fungi: screening of plant cell-wall degrading enzymes. African Journal of Microbiology research 5 (4) : 443-448.

Alemu K., 2014. Dynamics and management of major postharvest fungi diseases of mango fruits. Journal of Biology, Agriculture and Healthcare Vol. 4, N²2 : 2224-3208.

Alloue-Boraud W.A. M., Koffi L. B., Dadie A. T., Dje K. M., Ongena M., 2015. Utilisation de Bacillus subtilis GA1 pour la lutte contre les germes d'altération de la mangue en Côte D'ivoire. Journal of Animal \&Plant Sciences, Vol.25, Issue 3: 3954-3965.

Aquastat, 2005. Côte d'Ivoire: Géographie, climat et population. L'irrigation en Afrique en chiffreEnquête Aquastat. FAO : 10

Anonyme, 2013. Côte d'Ivoire, étude de collection dans le secteur agricole en Côte d'Ivoire. Rapport final. JICA JAICAF. : 19-30.

Anonyme, 2017. ONU Info. FAO: de plus en plus populaires, les fruits tropicaux représentent une opportunité pour les pays en développement. https://news.un.org/fr/audio/2017/11/1003791

Awa OC, Samuel O, Oworu O0, Sosanya 0, 2012. First report of fruit anthracnose in mango caused by Colletotrichum gloeosporioides in Southwestern Nigeria. International Journal of Scientific and Technology Research 1, 30-34.

Barnett HL et Hunter BB, 1972. Illustrated genera of imperfect fungi. 3rd Edition. Burgess Publishing Company : 200pp.

COLEACP, 2011. Lutte biologique et protection intégrée. Fondements de la protection des cultures, Manuels de formation du COLEACPPIP : 293p.

Dédi JKY, Allou KR, Otchoumou A, 2015. Inventaire de la flore fongique et identification des différents horizons du profil au sein de la bananeraie de Tadmait. International Journal of Biological and Chemical Science 9(3) : 1419-1430.

Diallo, 2016. Control of anthracnose (caused by Colletotrichum gloeosporiodes S.L.) on mango in Senegal by fungicides and biofungicides. Thesis submitted to the faculty of Virginia Polytechnic Institute and State Uniersity : 1-46. de leurs répartitions géographiques.

Diedhiou PM, M'Baye N, Drame A, Samb PI, 2007. Alteration of post harvest diseases of mango Mangifera indica through production practices and climatic factor. Full Length Research Paper. African Journal of Biotechnology 6 (9) : 1087-1094.

Djeugap JF, Kuiate JR, Fontem DA, 2009. Etat sanitaire post-récolte de la mangue commercialisée dans la ville de de Dschang et efficacité in vitro des huiles essentielles contre Colletotrichum gloeosporiodes Penz., agent causal de l'anthracnose. AFPP. gème conférence internationale sur les maladies des plantes Tours-8 et 9 décembre 2009: 571578.

Djeugap JF, Tsopmbeng NG, Keuete KE, Yaouba A, Serferbe S, 2015. Isolation and identification of fungi associated with avocado fruit from local markets of the west région of Cameroun. Inter J Agri Biosci, 4 (2) : 64-68.

Dongmo GZ, Djeugap JF, Fenohi N, Kenfack ND, Takuete $R$, Teguefouet $P, 2017$. Contribution à l'identification des champignons de postrécolte associés aux amandes de Ricinodendron heudelotii et Garcinia kola collectées dans les Hauts Plateaux de l'Ouest Cameroun. International Journal of Biologycal and Chemical Science 11 (4) : 1840-1850.

FAO, 2004. Technologies combinées de conservation des fruits et légumes. Manuel de formation. Service des technologies d'ingénierie agricole et alimentaire (AGST), Division des systèmes de soutien agricole (AGS). Département de l'agriculture, Archives de documents de la FAO.

FIRCA, 2014. Présentation des filières fruitières. La filière du Progrès $n^{\circ} 13 \mathrm{du}$ 1er trimestre 2014 : 4-5.

Kader AA et Yahia EM, 2011. Book Postharvest Biology and Technology of Tropical and subtropical fruits. Postharvest biology of tropical and subtropical fruits. Elhadi Yahia (Editor), 1'st édition woodhead publishing Quelato, Mexique : $534 p$.

Kaman P., 2013. Analyse des vulnérabilités aux catastrophes et des capacités de réponses en Côte d'Ivoire. Gouvernance du risque et 
diagnostic de vulnérabilité de la Côte d'Ivoire, Version final, UEMOA, RCl : 65 .

Kouadio KT, Agneroh TA, Pohé J, Tienebo EO, Ohoussou NILV, 2017. Inventaire des champignons pathogènes post-récolte de la Banane dessert «Cavendish » et évaluation de leur sensibilité à l'Azoxytrobine en Côte d'Ivoire. European Scientific Journal, 13 (21) : 26-43.

Kouamé G. K., Sorho F., Koné D., Bomisso L. E., Aké S., Yatty J., 2011. Activité pathologique comparée de deux isolats de Colletotrichum gloeosporioïdes (PENZ.) sur deux variétés de mangues (Mangifera indica L.). Agronomie Africaine 23 (1) : 33 - 41 .

Lakshmi BKM, Reddy PN, Prasad RD, 2011. Cross infection potential of Colletotrichum gloeosporioides Penz. Isolates causing anthracnose in subtropical fruit crops. Tropical agricultural research 22 (2) : 183-193.

Malik A, Iram S, Rasool A, 2016. Current statut of mango pre and post-harvest diseases with respect to environnemental factors. International Journal of Agronomy and Agricultural Research (IJAAR) 8 (4) : 101-110.

N'Guettia MY, Diallo HA, Kouassi N, Coulibaly F, 2013. Diversité morphologique et pathogénique des souches de Colletotrichum sp. responsable de l'anthracnose de la mangue en Côte d'Ivoire. Journal of Animal \& Plant Sciences, 18 (3) : 2775-2784

Nouar M., 2013. Evaluation du potentiel à l'exportation des fruits tropicaux. Programme d'Appui au Commerce et à l'Intégration Régional (PACIR), Appui Institutionnel et opérationnel pour l'amélioration du cadre des affaires et le renforcement de la compétitivité des exportations de la Côte d'Ivoire : 1-159.

PIP, Guide de bonnes pratiques phytosanitaires, 2013. Pour la mangue (Mangifera indica) issue de la production biologique. Programme PIP COLEACP, Belgique. $52 \quad \mathrm{pp}$. www.coleacp.org/pip.

Prusky D et Lichter A, 2007. Activation of quiescent infections by postharvest pathogens during transition from the biotrophic to the necrotrophic stage. In Minireview. Federation of European Microbiological Societies : 1-8.

Singh BK, Yadav SK, Verma A, 2017. Impact of diseases and their management in fruit crops and overwiew. J. Bio. Innov 65 : 749-760.
Spore, 2016. Agriculteurs connectés, Nouvelles perspectives pour l'agriculture. $180: 9$.

Telemans B, 2012. Technique de production de mangue pour l'exportation. RADHORT. FAO : 6-7.

Touré S, 2012. Etude nationale Mangue. La Côte d'Ivoire et le centre du commerce International : 1-27.

Vannière $\mathrm{H}$, Didier $\mathrm{C}$, Rey JY, Diallo TM, Kéita $\mathrm{S}$, Sangaré M, 2004. La mangue en Afrique de l'Ouest francophone: les systèmes de production et les itinéraires techniques. Fruits $59:$ 383-398.

Yaouba $A$ et Mpounze EGP, 2017. Isolation and pathogenicity evaluation of postharvest fungal of some fruits in Cameroun. International Journal of Environment, Agriculture and Biotechnology (IJEAB) 2 : 56-60.

Wharton SJ, Basu SP, Ashe HL, 2004. Smad affinity can direct distinct readouts of the embryonic extracellular Dpp gradient in Drosophila. Curr. Biol. 14(17): 1550-1558. 